\title{
Omisión de registros de causas maternas de muerte en Argentina: estudio observacional de alcance nacional
}

\author{
Edgardo Abalos, ${ }^{1}$ Mariana Duhau, ${ }^{2}$ Paola Escobar, ${ }^{2}$ María Letizia Fasola, ${ }^{2}$ Juliana Zoe Finkelstein, ${ }^{2}$ \\ José Luis Golubicki, ${ }^{3}$ Hugo Krupitzki, ${ }^{4}$ Élida Marconi, ${ }^{5}$ Adrián Santoro ${ }^{6}$ y Jorge Vinacur, ${ }^{7}$ \\ en representación del Estudio Colaborativo Argentino EORMM
}

Forma de citar

Abalos E, Duhau M, Escobar P, Fasola ML, Finkelstein JZ, Golubicki J, et al. Omisión de registros de causas maternas de muerte en Argentina: estudio observacional de alcance nacional. Rev Panam Salud Publica. 2019;43:e13. https://doi.org/10.26633/RPSP.2019.13.

RESUMEN

Palabras clave
Objetivo. Determinar la magnitud y características de la omisión del registro de causas maternas de muerte en Argentina y reestimar la razón de mortalidad materna (RMM) para el año 2014.

Métodos. Estudio de corte transversal. Revisión retrospectiva de registros médicos de mujeres en edad reproductiva que murieron por causas sospechosas de encubrir muertes maternas en instituciones del sector público, la seguridad social y privado en todas las jurisdicciones del país entre el 1 de enero y el 31 de diciembre de 2014. Se reclasificó la causa de muerte consignada en el Informe Estadístico de Defunción. Las medidas de resultado incluyeron el porcentaje de omisión del registro, la estructura de causas, el lugar, el momento de la muerte en relación con el proceso reproductivo y la edad gestacional. Se reestimó la RMM en base a los resultados.

Resultados. Se analizaron 969 registros médicos (82,4\%) de una muestra de 1176 casos. Se identificaron 60 casos donde se omitió la causa materna de muerte (48 muertes maternas, 12 muertes maternas tardias). El porcentaje de omisión fue del 14,2\% para las muertes maternas y del 33,3\% para las muertes maternas tardías. La nueva RMM estimada para el año 2014 osciló entre 43,3 y 47,2 muertes por cada 100000 nacidos vivos.

Conclusiones. La omisión de registro de causas maternas de muerte en Argentina podría ser menor a la reportada por agencias internacionales. Existen diferencias de omisión entre las regiones. Se necesitan esfuerzos que enfaticen la importancia del llenado correcto de los certificados de defunción.

Omisiones de registro; muerte materna; estadísticas vitales; estudio observacional; Argentina.
Centro Rosarino de Estudios Perinatales (CREP), Rosario, Argentina. Enviar correspondencia a Edgardo Abalos, edgardoabalos@crep.org.ar

2 Dirección Nacional de Maternidad, Infancia y Adolescencia, Secretaría de Salud de la Nación, Argentina.

Sociedad Argentina de Terapia Intensiva (SATI), Ciudad Autónoma de Buenos Aires, Argentina.

Centro de Educación Médica e Investigaciones Clínicas - Consejo Nacional de Investigaciones
Científicas y Técnicas (CEMIC-CONICET), Ciudad Autónoma de Buenos Aires, Argentina.

Centro Argentino de Clasificación de Enfermedades (CACE) - Centro Colaborador para la Familia de Clasificaciones Internacionales de la OMS en Argentina, Secretaría de Salud de la Nación, Argentina. Dirección de Estadísticas e Información de Salud Secretaría de Salud de la Nación, Argentina. Sociedad de Obstetricia y Ginecología de Buenos Aires (SOGIBA), Ciudad Autónoma de Buenos Aires, Argentina.
La Organización de las Naciones Unidas reconoce la mortalidad materna como un problema de salud pública y derechos humanos, y subraya que afecta el derecho de las mujeres y las niñas a la vida, la salud, la equidad y la no discriminación, a disfrutar de los avances del conocimiento científico y al más alto nivel posible de salud (1).

Este es un artículo de acceso abierto distribuido bajo los términos de la licencia Creative Commons Attribution-NonCommercial-NoDerivs 3.0 IGO, que permite su uso, distribución y reproducción en cualquier medio, siempre que el trabajo original se cite de la manera adecuada. No se permiten modificaciones a los artículos ni su uso comercial. Al reproducir un artículo no debe haber ningún indicio de que la OPS o el artículo avalan a una organización o un producto específico. El uso del logo de la OPS no está permitido. Esta leyenda debe conservarse, junto con la URL original del artículo. 
FIGURA 1. Flujograma de búsqueda e identificación de casos (Estudio de Omisión de Registro de Causas Maternas de Muerte, EORMM)

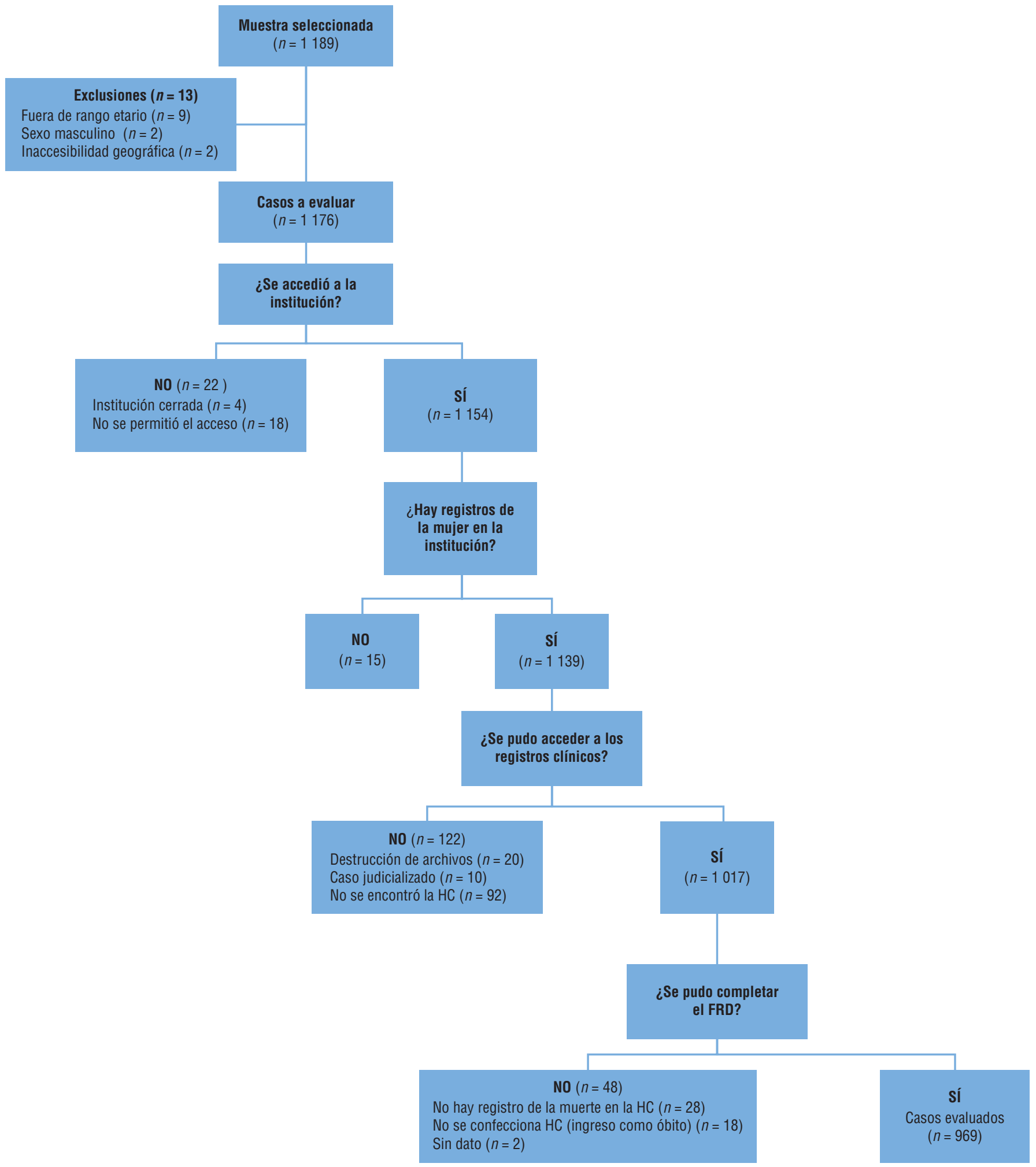

La Organización Mundial de la Salud (OMS) define la muerte materna como la muerte de una mujer durante el embarazo o dentro de los 42 días posteriores a su finalización, cualquiera sea su duración y ubicación, por cualquier causa relacionada o agravada por el embarazo o su cuidado, pero no por causas accidentales o incidentales (2). Define la razón de mortalidad materna (RMM) como el número de defunciones maternas por cada 100000 nacidos vivos.

Sin embargo, incluso en países con estadísticas vitales confiables, existen dificultades para identificar todas las 
muertes maternas que ocurren en sus regiones (3-5). Esta situación llevó a la creación del Grupo Interinstitucional de Estimación de la Mortalidad Materna (MMEIG, por sus siglas en inglés), una iniciativa integrada por la OMS, el Fondo de las Naciones Unidas para la Infancia (UNICEF, por sus siglas en inglés), el Fondo de Población de las Naciones Unidas (FPNU) y el Banco Mundial, responsable de realizar estimaciones de la RMM en función de la calidad de la información recopilada por cada país, las tendencias de la población y la existencia de estadísticas vitales (6). Para la Argentina, desde 1990, el MMEIG aplicaba un factor de corrección de 1,5 (es decir, un incremento del $50 \%$ en la RMM) a las muertes maternas registradas por el país. El denominador, por otro lado, se basaba en estimaciones de la Comisión Económica para América Latina y el Caribe (CEPAL) que subestiman el número de recién nacidos registrados en Argentina. Estas diferencias (aumento en el numerador y disminución en el denominador) resultaron en un aumento en la RMM estimada. En 2013, la RMM publicada por el MMEIG para Argentina fue de 69 por cada 100000 nacidos vivos, sustancialmente más alta que la registrada y publicada por el Ministerio de Salud de la Nación (de 32 por cada 100000 nacidos vivos) (7). Sin embargo, a partir del año 2015, el MMEIG utiliza un enfoque metodológico mejorado que prioriza en cada país los datos de mayor calidad para generar las estimaciones (8). Mediante el uso de esta nueva metodología, la estimación de la RMM en la Argentina para el año 2013 fue de 54 por cada 100000 nacidos vivos (9).

Estudios previos dirigidos a conocer la magnitud de la omisión de registro de causas maternas de muerte (muertes de mujeres en edad fértil registradas en las estadísticas vitales, pero cuya causa básica de defunción fue clasificada como no materna) en nuestro país arrojaron resultados disímiles (10-12). Por lo tanto, el Ministerio de Salud de la Nación a través de la Dirección Nacional de Maternidad, Infancia y Adolescencia, la Dirección de Estadísticas e Información de Salud, el Centro Argentino de Clasificación de Enfermedades, junto con sociedades científicas (Sociedad de Obstetricia y Ginecología de Buenos Aires, Sociedad Argentina de Terapia Intensiva) y centros de investigación (Centro de Educación Médica e Investigaciones Clínicas,

CUADRO 1. Muertes maternas identificadas en el estudio según momento de la gestación, evento obstétrico, y características del sitio de internación

\begin{tabular}{llrr}
\hline Momento de la gestación & $\mathrm{n}$ & $\%$ \\
\hline Durante el embarazo & Antes de las 20 semanas de gestación & 17 & 28,3 \\
$\mathbf{n}=\mathbf{2 4}$ & Después de las 20 semanas de gestación & 5 & 8,3 \\
& Intraparto & 2 & 3,3 \\
Posterior al embarazo & Posparto (0 a 7 días) & 10 & 16,7 \\
$\mathbf{n = 3 6}$ & Posparto (8 a 42 días) & 14 & 23,3 \\
& Posparto (mayor a 42 días) y menor a 1 año & 12 & 20,0 \\
Total & & $\mathbf{6 0}$ & $\mathbf{1 0 0}$ \\
\hline Establecimiento de salud & $\mathbf{N}$ & $\%$ \\
$\quad$ Establecimiento de salud pública & 49 & 81,7 \\
$\quad$ Establecimiento privado o de seguridad social & & 11 & 18,3 \\
Total & & $\mathbf{6 0}$ & $\mathbf{1 0 0}$ \\
\hline Lugar de la defunción & $\mathbf{N}$ & $\%$ \\
$\quad$ Sala de obstetricia/ginecología & 1 & 1,7 \\
Quirófano & 3 & 5,0 \\
Guardia general/clínica médica & 7 & 11,7 \\
$\quad$ Unidad de terapia intensiva/unidad coronaria & 44 & 73,3 \\
$\quad$ Otros & 0 & 0,0 \\
$\quad$ Se desconoce/sin datos & 5 & 8,3 \\
Total & & $\mathbf{6 0}$ & $\mathbf{1 0 0}$ \\
\hline
\end{tabular}

Centro Rosarino de Estudios Perinatales) realizaron un estudio en las 24 jurisdicciones del país (las 23 provincias y la Capital Federal) con el objetivo de: 1) conocer la magnitud de la omisión del registro de causas maternas de muerte en defunciones institucionales de mujeres en edad fértil identificadas primariamente como muertes por causas no maternas, en general y por jurisdicción, 2) describir las causas y las características de las instituciones donde ocurrieron, y 3) reestimar la RMM para el año 2014. (Estudio de Omisión de Registro de Causas Maternas de Muerte, EORMM).

\section{MATERIALES Y MÉTODOS}

Estudio observacional, de corte transversal. Se revisaron y analizaron los registros clínicos de las muertes de mujeres de 10 a 49 años registradas en Argentina entre el 1 de enero y el 31 de diciembre de 2014, ocurridas en instituciones de los sectores público, de seguridad social y privado. Se excluyeron las muertes no institucionales, pues las instituciones de salud no suelen realizar registros clínicos si el evento ocurre fuera de ellas. La identificación de casos se basó en la causa básica de muertes sospechosas por la cual se ocultan muertes maternas registradas en el Informe Estadístico de Defunción (IED). La preparación de esta lista de causas surge del Protocolo para la Mejora de la
Información sobre Mortalidad Materna de la Organización Panamericana de la Salud (OPS) (13), adaptado por una comisión de expertos a las modalidades de registro en Argentina. ${ }^{8}$ En la muestra se incluyeron todos los departamentos que concentraron, en cada jurisdicción, 10 o más casos de defunciones institucionales sospechosas de ocultar muertes maternas. En las jurisdicciones donde ningún departamento alcanzó los 10 casos, se incluyeron todos los departamentos que presentaron al menos cinco casos. En aquellas jurisdicciones donde ningún departamento tuvo más de cinco casos, se incluyeron todos los departamentos $(P=1)$. Para todos los departamentos que no quedaron incluidos según el criterio anterior se seleccionó una muestra aleatoria sistemática del $20 \%$, con selección proporcional a la cantidad de defunciones de causas sospechosas, por cada jurisdicción.

La fuente principal de datos fueron los registros clínicos de la última hospitalización de la mujer, los libros de guardia de las salas de emergencias y cualquier otro informe de hospitalización disponible en la institución donde se consignó la muerte.

La medida de resultado principal fue el porcentaje de omisión de registro de causa materna de muerte, definido como la proporción de muertes

\footnotetext{
Listado de causas adaptado, disponible para el lector interesado que lo solicite al autor de correspondencia.
} 
maternas identificadas por el estudio entre el número total estimado de muertes maternas (aquellas identificadas por el estudio más las informadas en las estadísticas vitales). Los resultados secundarios incluyeron la estructura de causas de muertes maternas entre los casos identificados y el registrado en su momento en el IED, el número de muertes por jurisdicción, por sector administrativo del establecimiento (público, seguridad social y privado), y por unidad hospitalaria donde ocurrió la muerte (sala de emergencia, sala de operaciones, unidad de obstetricia o ginecología, unidad de medicina interna o general, unidad de cuidados intensivos $\mathrm{u}$ otros). También se analizaron el momento de la muerte con respecto al proceso reproductivo (anteparto, intraparto, aborto, postparto de 0 a 7 días, posparto de 8 a 42 días o posparto entre los 42 días y un año) y la edad gestacional del evento obstétrico (antes o después de las 20 semanas de gestación) al momento de la muerte. Por último, se recalculó la RMM publicada en el Anuario de Estadísticas Vitales para el año 2014; para esto, se añadió al numerador el número de muertes maternas omitidas identificadas en el estudio, con su respectiva dispersión.

Las frecuencias se describieron como porcentajes con sus intervalos de confianza de 95\% (IC95\%) y las variables continuas, como media o mediana según su distribución y dispersión. Para asegurar la representatividad de las regiones más pequeñas, se aplicó un factor de expansión para cada una de las unidades de muestreo, con el objeto de restituir a cada provincia y departamento el peso real de los casos evaluados en el universo de casos a evaluar. Para expandir la muestra en función de

CUADRO 2. Número de muertes maternas publicado en el Anuario de Estadísticas Vitales, número de muertes maternas identificadas en el estudio, total estimado de muertes maternas (registradas y omitidas como causas maternas) y porcentaje de muertes no registradas como muertes maternas, según jurisdicción de residencia, 2014

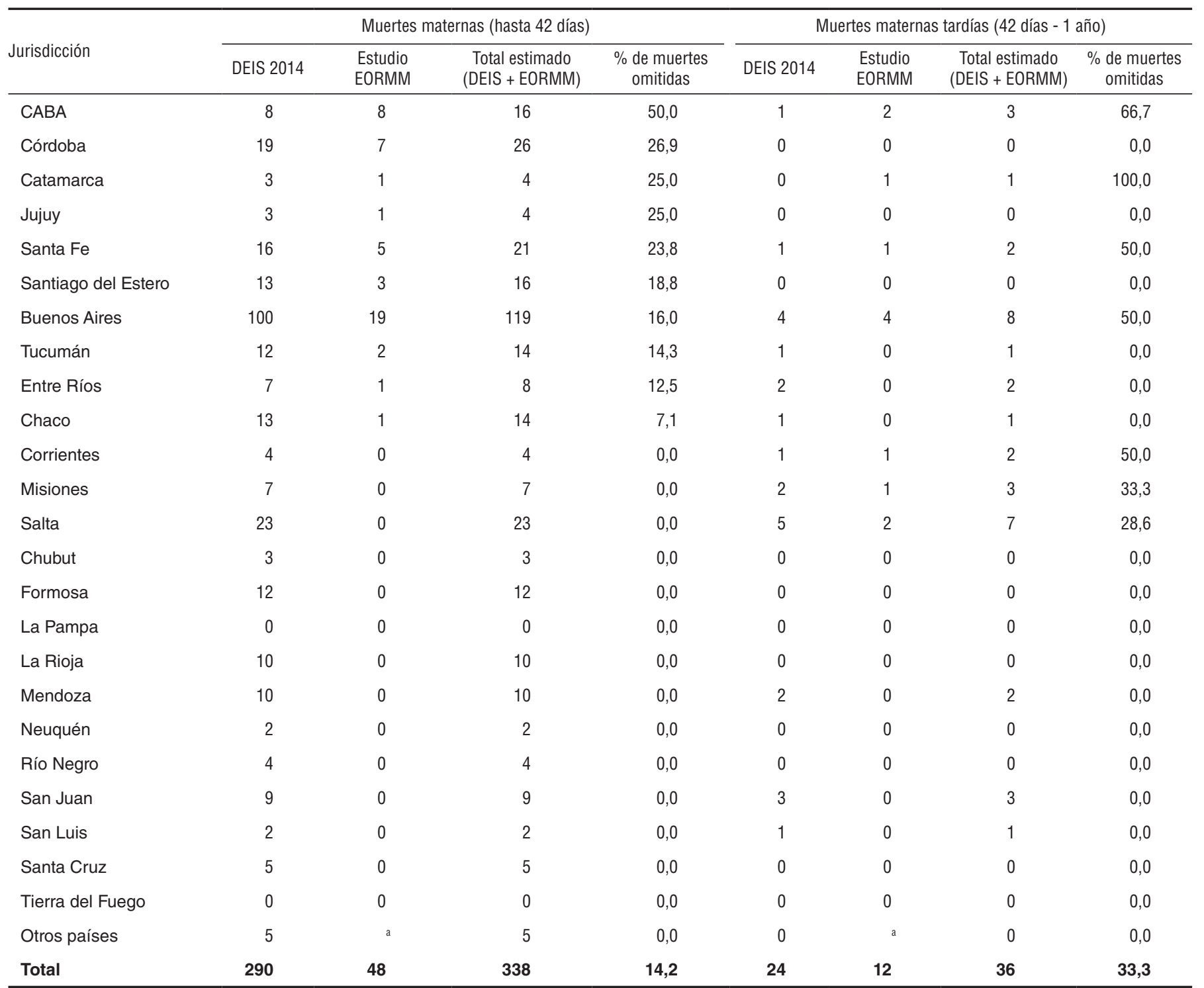

C.A.B.A., Ciudad Autónoma de Buenos Aires.

aEl estudio EORMM no realizó búsqueda activa de casos en otros países. 
las unidades de muestreo agregadas en departamentos, se obtuvo un coeficiente y se procedió en cada una de ellas como si fueran independientes. El valor del expansor se interpretó como el número total de casos de muertes sospechosas de ocultar muertes maternas que es representado por cada caso de la muestra seleccionada.

Los datos se recolectaron en un formulario de recolección de datos (FRD) prediseñado, se ingresaron en una base de datos electrónica y se analizaron utilizando el paquete estadístico STATA $12.0^{\circledR}$, supervisado por la Coordinación General y la Comisión Asesora Nacional.

El protocolo del estudio fue aprobado por el Comité de Ética en Investigación del CEMIC ( $\mathrm{N}^{\circ} 1$ 038, 13 de junio de 2016) y los Comités Provinciales de Bioética y los Comités de Bioética y/o Docencia e Investigación de las instituciones participantes.

\section{RESULTADOS}

En el año 2014 se registraron en el país 1483 muertes institucionales de mujeres de 10 a 49 años, en las que al menos una de las 55 causas sospechosas de ocultar muertes maternas se consignó en el IED. Se seleccionó una muestra de 1189 casos $(80,2 \%)$ de acuerdo con los criterios y estratos antes especificados en las 24 jurisdicciones del país. Se excluyeron dos casos por dificultad geográfica para acceder a las instituciones. Luego se identificaron en los registros clínicos once casos que no cumplían con los criterios de elegibilidad: nueve debido a edades fuera del rango (menores de 10 años o mayores de 49), y dos por tratarse de muertes de personas de sexo masculino. La muestra final estuvo constituida por 1176 casos.

Entre agosto y noviembre de 2016 se buscaron los casos elegibles en 394 centros de salud correspondientes a 76 departamentos de las 24 jurisdicciones del país. No se pudo acceder a las instituciones en 22 casos (1,9\%). Cuatro instituciones (4 casos) ya no existían, y en 12 instituciones (18 casos) no se obtuvo aprobación para acceder a los registros por parte de las autoridades o los comités de ética locales. De los casos restantes, en $15(1,3 \%)$ no había registros de que las mujeres fueran atendidas en el establecimiento, y en $122(10,4 \%)$ no se pudo acceder a los documentos médicos o registros hospitalarios. Entre estos últimos, en 20 casos (10 instituciones), las historias clínicas fueron destruidas en incendios o inundaciones de los archivos; en 10 casos (siete instituciones), los registros médicos no estaban disponibles debido a litigios judiciales; en los 92 casos restantes, las

CUADRO 3. Omisión de registro de causa materna de muerte en Argentina. Causas re-clasificadas por el estudio EORMM y originalmente reportadas en el IED

\begin{tabular}{|c|c|c|c|}
\hline Causas según el estudio EORMM-Argentina & $\mathrm{N}$ & Causas originalmente reportadas en el IED & $\mathrm{N}$ \\
\hline Directas & 32 & & \\
\hline \multirow[t]{4}{*}{ Aborto $(005.5,006.0,006.5)$} & 6 & Sepsis & 1 \\
\hline & & Edema pulmonar & 2 \\
\hline & & Shock & 1 \\
\hline & & No especificada & 2 \\
\hline Embarazo molar (001.9) & 1 & Falla cardíaca & 1 \\
\hline Embarazo ectópico (000.9) & 2 & No especificada & 2 \\
\hline \multirow[t]{6}{*}{ Hipertensión/eclampsia (014.1, 015, 015.2, 015.9) } & 6 & Anoxia cerebral & 1 \\
\hline & & Hemorragia intracraneal & 1 \\
\hline & & Accidente cerebrovascular & 1 \\
\hline & & Shock & 1 \\
\hline & & Miocardiopatía & 1 \\
\hline & & No especificada & 1 \\
\hline Tromboembolismo pulmonar (088.2) & 2 & Embolia pulmonar & 2 \\
\hline \multirow{3}{*}{$\begin{array}{l}\text { Hemorragia/desprendimiento normoplacentario } \\
(045.9,072.1,072.3)\end{array}$} & 4 & Puerperio & 1 \\
\hline & & Insuficiencia hepática & 1 \\
\hline & & No especificada & 2 \\
\hline \multirow[t]{7}{*}{ Otras causas directas $(036.4,074.3,075.4,090.3)$} & 9 & Convulsión & 1 \\
\hline & & Edema cerebral & 2 \\
\hline & & Hemorragia intraencefálica & 1 \\
\hline & & Sepsis & 2 \\
\hline & & Neumonía & 1 \\
\hline & & Miocardiopatía & 1 \\
\hline & & Embolia pulmonar & 1 \\
\hline Indirectas & 16 & & \\
\hline \multirow{10}{*}{$\begin{array}{l}\text { Otras causas indirectas }(023.4,098.0,099.4 \text {, } \\
\text { 099.5, 099.6, } 099.8\end{array}$} & 13 & Hemorragia subaracnoidea & 1 \\
\hline & & Hemorragia intraencefálica & 1 \\
\hline & & Hemorragia intracraneal & 1 \\
\hline & & Otra causa cerebrovascular & 1 \\
\hline & & Coagulación intravascular diseminada & 1 \\
\hline & & Embolia pulmonar & 1 \\
\hline & & Falla cardíaca & 1 \\
\hline & & Neumonía & 4 \\
\hline & & Peritonitis & 1 \\
\hline & & Insuficiencia renal & 1 \\
\hline \multirow[t]{2}{*}{ VIH/SIDA (B22.0, B24) } & 2 & Sepsis & 1 \\
\hline & & Neumonía & 1 \\
\hline $\begin{array}{l}\text { Otras enfermedades y afecciones específicas que } \\
\text { complican el embarazo, el parto y el puerperio (099.8) }\end{array}$ & 1 & No especificada & 1 \\
\hline Tardías & 12 & & \\
\hline \multirow[t]{9}{*}{$(014.1,096,099.4)$} & 12 & Sepsis & 2 \\
\hline & & Neumonía & 3 \\
\hline & & Hipertensión cerebral & 1 \\
\hline & & Hemorragia subaracnoidea & 1 \\
\hline & & Hemorragia intraencefálica & 1 \\
\hline & & Accidente cerebrovascular & 1 \\
\hline & & Embolia pulmonar & 1 \\
\hline & & Falla cardíaca & 1 \\
\hline & & Insuficiencia renal & 1 \\
\hline
\end{tabular}

Nota: Ios números entre paréntesis corresponden a los códigos de la Clasificación Internacional de Enfermedades (CIE-10). 
historias clínicas no pudieron ubicarse en los archivos.

Del total de casos en los que se obtuvo acceso a registros hospitalarios, el FRD no pudo completarse en $48(4,1 \%)$. En 18 de estos casos no se confeccionaron registros médicos en la institución debido a que las mujeres murieron antes de llegar a ella (a pesar de que en el IED se registró la muerte como institucional), en 28 no hubo registros de la última hospitalización correspondiente a la muerte, y en los otros dos el motivo no fue informado. Por lo tanto, se extrajeron datos de los registros médicos de 969 mujeres, lo que corresponde al 82,4 \% de la muestra (figura 1).

La cobertura (definida como el número de casos evaluados sobre el total de casos a evaluar) fue alta en todo el país. Solo nueve jurisdicciones $(9 / 24)$ tuvieron una cobertura inferior al $80 \%$, y en solo cuatro esta fue inferior al $75 \% .{ }^{9}$

De las muertes analizadas, 60 correspondieron a muertes asociadas al embarazo, parto/aborto y puerperio. Cuarenta y ocho de estas muertes $(80 \%)$ ocurrieron durante el embarazo o hasta los cuarenta y dos días del parto o aborto (muertes maternas propiamente dichas), las 12 restantes correspondieron a muertes maternas tardías. Más del 80\% de los establecimientos de salud donde se identificaron muertes maternas pertenecen al sector público. La mayoría de las muertes ocurrieron en las unidades de cuidados intensivos o cuidados coronarios (cuadro 1).

En el cuadro 2 se muestran los casos identificados de muertes maternas y muertes maternas tardías según la jurisdicción de residencia, en comparación con las reportadas en el Anuario de Estadísticas Vitales del año 2014 y la proporción estimada de omisión de registro. El porcentaje de muertes no reportadas originalmente como muertes maternas y muertes maternas tardías fue de $14,2 \%$ y del $33,3 \%$, respectivamente. La mayoría de las muertes maternas ocurrieron en la Ciudad Autónoma de Buenos Aires (CABA), en las provincias de Buenos Aires y Santiago del Estero y en los grandes centros urbanos de las provincias más pobladas (Córdoba y Santa Fe). En otras dos provincias (Catamarca y Jujuy), la proporción de muertes no registradas

Tabla de cobertura por jurisdicción disponible para el lector interesado que lo solicite al autor de correspondencia.

FIGURA 2. Estructura de causas de muertes maternas, publicadas por DEIS 2014 y corregidas de acuerdo a EORMM-Argentina 2017

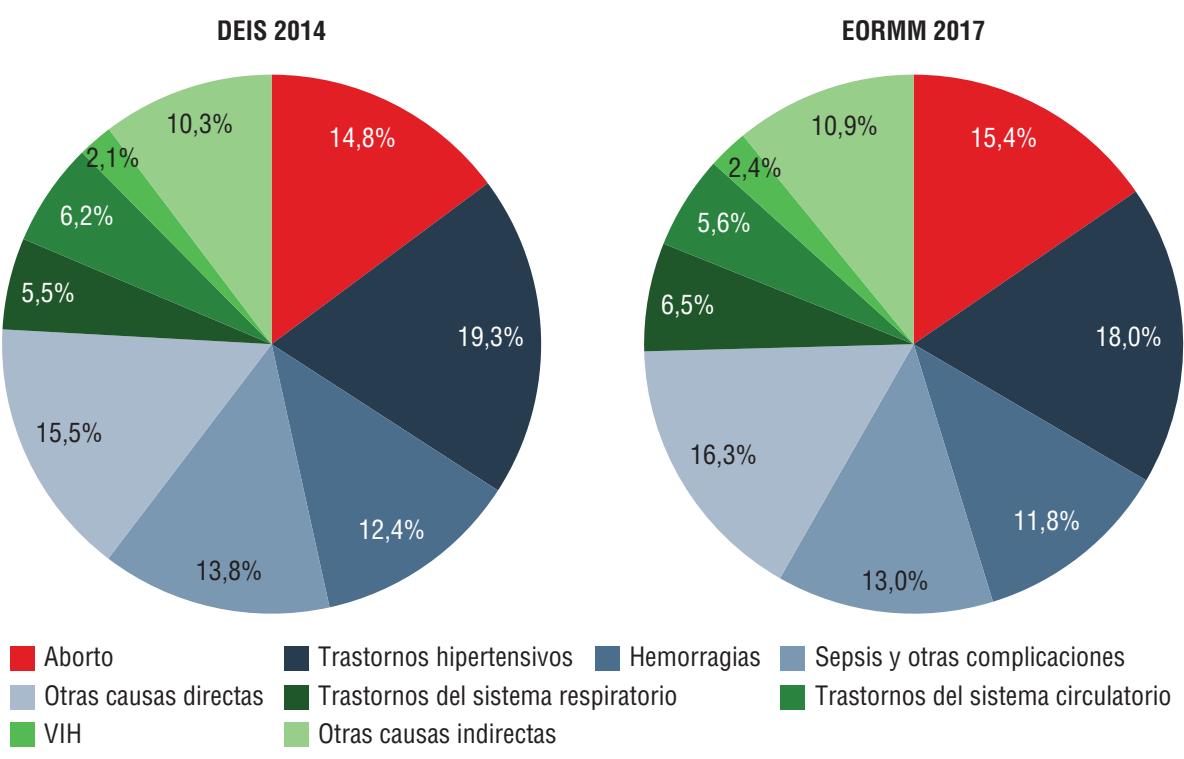

VIH, virus de la inmunodeficiencia humana.

originalmente como maternas también fue alta, pero estas cifras, así como las muertes tardías pueden verse distorsionadas debido al pequeño número de casos.

En nueve de las 48 muertes maternas, se identificó al aborto como causa principal (incluidos dos embarazos ectópicos), 23 se debieron a otras causas directas y 16 a causas indirectas. En el cuadro 3 se muestra la distribución de las muertes maternas identificadas por el investigador en comparación con la causa básica registrada en el IED.

En el año 2014 se registraron, en el Anuario de Estadísticas Vitales de la República Argentina, 777012 nacidos vivos y 290 muertes maternas, lo que arrojó una RMM de 37 por cada 100000 nacidos vivos. En nuestro estudio hemos identificado 48 muertes maternas adicionales. El valor expandido en función de las unidades de muestreo agregadas en departamentos al universo de las muertes institucionales de mujeres de 10 a 49 años con causas sospechosas de ocultar muertes maternas, asciende a 62 (IC95\%: 47-77). Al sumar estas muertes a las oficialmente publicadas, la RMM estimada para la Argentina en el año 2014 asciende a 45,3 (IC 95\%: 43,3-47,2) por cada 100 000 nacidos vivos.

La estructura de las causas de muerte, luego de incorporar los 48 casos identificados por el estudio, no sufrió variaciones significativas (figura 2).

\section{DISCUSIÓN}

Este es el primer estudio que evalúa la omisión del registro de causas maternas de muerte con alcance nacional. El mismo resulta de la colaboración entre el Ministerio de Salud de la Nación, sociedades científicas y centros de investigación. Se realizó en instituciones de salud de todas las jurisdicciones del país, con una metodología única y de manera simultánea.

Por cuestiones de logística y viabilidad, se planteó la dificultad de utilizar otras técnicas para la detección de los casos (como las encuestas confidenciales) o de analizar la totalidad de los casos sospechosos en todo el territorio nacional. Para la obtención de la muestra, se aplicaron los criterios de estratificación descritos para la selección de los departamentos según el número de casos en cada jurisdicción. Sin embargo, los departamentos con mayor número de casos coinciden con los grandes conglomerados urbanos, por lo cual limitar el estudio a estos departamentos hubiera dejado invisibilizada, en esas jurisdicciones, a las poblaciones más pequeñas o remotas, tal vez con mayores dificultades de acceso al sistema de salud, vulnerabilidades económicas y sociales. La adición de una muestra aleatoria sistemática de $20 \%$, con selección proporcional a la cantidad de defunciones, permitió garantizar la representatividad de los 
departamentos con escaso número de casos pertenecientes a las provincias que albergan estos grandes centros urbanos. La muestra seleccionada se expandió, además, en función de las unidades de muestreo agregadas en departamentos; de esta manera se restableció el peso de cada caso evaluado al total de muertes institucionales de mujeres de 10 a 49 años cuya causa registrada en el IED fuera sospechosa de ocultar muertes maternas.

En 17,3\% de los casos no se pudo acceder a los registros clínicos de las mujeres, la mayoría de las veces por incapacidad de las instituciones de custodiarlos (incendios, inundaciones, pérdida total o parcial de registros), lo que podría afectar los resultados y constituir una limitación del estudio. Sin embargo, no encontramos un patrón sistemático en estas pérdidas, por lo que aún ante esta circunstancia consideramos que la cobertura del estudio fue alta, y al incluir instituciones públicas y privadas los resultados son generalizables. Estudios previos realizados en el país informaron resultados disímiles. En 1985, la Dirección Nacional de Maternidad e Infancia junto con la Dirección de Estadísticas e Información en Salud realizaron un estudio en la Ciudad Autónoma de Buenos Aires y la provincia de Córdoba y se encontró una omisión en el registro de muertes maternas de 53\% (10). En 2004, el Centro de Estudios de Estado y Sociedad junto al Centro Latinoamericano de Perinatología constataron, en las provincias del nordeste, noroeste $y$ Cuyo, una omisión de $9 \%$ para muertes maternas y de $13,5 \%$ para muertes maternas tardías (11). En 2008, la Sociedad de Ginecología y Obstetricia de Buenos Aires encontró una omisión del registro de defunciones maternas de $66 \%$ en la Ciudad Autónoma de Buenos Aires (12). Sin embargo, estos estudios fueron restringidos en la selección de los lugares, en diferentes períodos y con distintas metodologías, por lo que sus resultados no pueden generalizarse a todo el país. Sin embargo, los datos del estudio EORMM desagregados por jurisdicción presentados en el cuadro 2 son similares a los arrojados por estos estudios.

También se observa disparidad en estudios realizados en otros países. Una comparación de las muertes maternas detectadas mediante consultas confidenciales con las registradas en las estadísticas vitales en cinco países europeos entre 2000 y 2004 mostró porcentajes de subestimación entre 17,1\% en Francia y $87,5 \%$ en Eslovenia (14). Un estudio en Austria informó subregistros de 38\% para el período 1980-1998 (15). Las muertes maternas mal informadas y mal clasificadas también arrojaron resultados diferentes, como $28 \%$ en México (16), 37\% y $48 \%$ en las ciudades brasileñas de Campinas (17), y Pernambuco (18), respectivamente, 58-65\% en Taiwán $(19,20)$ o $76 \%$ en Jamaica $(21)$.

Nuestros datos sugieren que el porcentaje de omisiones en el registro de causas maternas de muerte a nivel nacional es menor al estimado por el MMEIG (14,2\% versus 50\%), aunque existen variaciones regionales, sobre todo en los grandes entornos urbanos. Un tercio de las jurisdicciones mostraron valores por encima de la media nacional, entre las que se destacan la Ciudad Autónoma de Buenos Aires (50\%), Córdoba $(26,9 \%)$, Catamarca y Jujuy $(25,0 \%)$ y Santa Fe $(23,8 \%)$. La inclusión, en algunas Oficinas Provinciales de Estadística, del pareo sistemático de la información sobre las causas de muerte consignada en el IED con lo registrado en los documentos clínicos de los establecimientos de salud podría explicar el bajo porcentaje de omisiones de causas maternas detectadas en algunas provincias. Esta iniciativa dirigida a reducir la omisión de la condición materna en IED ha sido exitosamente implementada en México (22), donde se compara la información de las causas de muerte registradas en el IED con los datos de las historias clínicas de las mujeres que murieron en la edad reproductiva por causas sospechosas de ocultar muertes maternas, ya sea por muestreo o en la totalidad de las muertes, como parte del sistema de reparaciones de las estadísticas vitales. Para facilitar la búsqueda intencional, en el año 2004 Argentina incorporó en el IED una pregunta sobre el estado de embarazo en los últimos 12 meses para todas las mujeres en edad fértil. Sin embargo, la cobertura de estos datos en el IED aún es escasa.

Con respecto al momento de la muerte en relación con el embarazo, es interesante observar que en 17 casos identificados por nuestro estudio (28,3\%) la muerte se relacionó con gestaciones de más de 20 semanas, o muy cerca del evento obstétrico (hasta 7 días), y no fueron identificados originalmente en el
IED. Esto puede reflejar la falta de capacitación del profesional de salud que completa el IED. En relación con las causas de muerte, a diferencia de estudios previos en los que hubo una mayor concentración de algunas causas particulares entre los casos no registrados $(10,11)$, las causas de muerte materna detectadas en este estudio se distribuyeron en forma homogénea, sin alterar la estructura de causas publicadas por el Ministerio de Salud. El aborto, una de las principales causas de mortalidad materna en Argentina durante los últimos 20 años, fue la condición más frecuente entre las causas directas detectadas. De acuerdo con otros estudios $(5,16)$, el accidente cerebrovascular y otras enfermedades cerebrovasculares fueron a menudo informados en el IED y, cuando se reclasificaron, se distribuyeron por igual entre las causas directas e indirectas de muerte materna. La enfermedad cerebrovascular no es una causa común de morbilidad y mortalidad entre mujeres en edad reproductiva, por lo que su informe como causa de muerte debe llamar la atención para investigar de manera activa un posible vínculo con el embarazo o el puerperio.

Aún existen situaciones que facilitan la omisión del estado grávido-puerperal en los registros de muertes, como son el desconocimiento de la condición de embarazo en embarazos muy precoces, o la dificultad de recuperar información cuando la muerte se aleja del evento obstétrico. Esto es en particular importante en unidades no obstétricas, como las salas de emergencia o unidades de cuidados intensivos, donde la gran cantidad de casos y su complejidad clínica dificultan la certificación de defunción (23). En nuestro estudio, $81 \%$ de las 60 muertes maternas detectadas ocurrieron en establecimientos de salud pública, y la mayoría en los servicios de unidades de cuidados intensivos, unidades coronarias y salas de emergencia.

La baja calidad en los registros de causas de muerte no solo afecta las estadísticas de mortalidad materna, sino todas las cifras de mortalidad (24). La falta de conocimiento o capacitación del personal de salud sobre la importancia estadística y para la salud pública del llenado correcto de los IED, incluido el registro de la situación de embarazo o puerperio en la certificación de la muerte es un problema grave, por lo que la OMS lanzó un llamado a los profesionales de la salud, 
los administradores y los decisores en políticas de salud para establecer medidas urgentes para enmendar esta situación (25).

\section{CONCLUSIONES}

El porcentaje de omisión de registro de causas maternas de muerte en Argentina fue inferior al reportado por las agencias internacionales. Además, existe heterogeneidad entre las jurisdicciones y los tipos de establecimientos de salud en la magnitud de la omisión. Por lo tanto, se debe enfatizar la importancia del llenado correcto de los registros clínicos y estadísticos, en particular el IED, tanto en la formación de pregrado como de posgrado. Se necesita capacitación enfocada en servicios específicos, en especial aquellos relacionados con cuidados críticos y salas de emergencia. El papel de las sociedades científicas, las asociaciones profesionales y las universidades en las actividades de capacitación también es crucial. Los encargados de la toma de decisiones y los gestores deben implementar sistemas de evaluación periódicos para la calidad de estos registros a fin de generar datos confiables para la toma de decisiones y las políticas de salud.

Agradecimientos. Los participantes del Estudio EORMM-Argentina agradecen a todas las autoridades nacionales y provinciales que brindaron apoyo durante el desarrollo del estudio, a los miembros de la Comisión Nacional Asesora, las agencias de cooperación internacional, los centros de investigación y las sociedades científicas que acompañaron el proceso de esta investigación. Un reconocimiento especial para los equipos provinciales locales, a las autoridades y trabajadores de los establecimientos de salud participantes, y a los supervisores e investigadores involucrados en el estudio, por su compromiso.

Financiamiento. Este trabajo fue financiado por la Dirección Nacional de Maternidad, Infancia y Adolescencia (DINAMIA) del Ministerio de Salud de la Nación de Argentina, con el apoyo técnico del Departamento de Estadística e Información en Salud (DEIS).
Los miembros de la coordinación central eran empleados en diferentes departamentos del ministerio o contratados en forma temporal para este estudio. Los equipos de las oficinas de estadísticas de maternidad, infancia y salud, y las direcciones de hospitales pertenecían al gobierno provincial y no se hicieron otros copagos por este trabajo. Investigadores y supervisores fueron contratados también en forma temporal para la realización del estudio. La participación de los miembros de la Comisión Nacional Asesora fue honoraria.

Conflicto de intereses. Mariana Duhau, María Letizia Fasola, Juliana Zoe Finkelstein, Élida Marconi y Adrián Santoro pertenecen al Ministerio de Salud de la Nación. Los otros miembros del equipo de revisión no tienen conflictos de intereses para declarar.

Declaración. Las opiniones expresadas en este manuscrito son responsabilidad del autor y no reflejan necesariamente los criterios ni la política de la RPSP/PAJPH y/o de la OPS.

\section{REFERENCIAS}

1. Asamblea General Naciones Unidas. Consejo de Derechos Humanos. Resolución A/HRC/11/L.16. Promoción y protección de todos los derechos humanos y de los derechos civiles, políticos, económicos sociales y culturales, incluido el derecho al desarrollo, 16 de junio de 2009.

2. International Statistical Classification of Diseases and Related Health Problems 10th Revision, Version 2016. (ICD-10). World Health Organization. Disponible en: http://apps.who.int/classifications/ icd 10 / browse / Content/statichtml / ICD10Volume2_en_2016.pdf Acceso el 15 de agosto de 2018.

3. Bouvier-Colle MH1, Varnoux N, Costes P, Hatton F. Maternal mortality in France. Frequency and reasons for its underestimation in the statistics of medical cause of death. Group of Experts on Maternal Mortality. J Gynecol Obstet Biol Reprod (Paris). 1991;20(7):885-91.

4. Turner LA1, Cyr M, Kinch RA, Liston R, Kramer MS, Fair M, et al; Maternal Mortality and Morbidity Study Group of the Canadian Perinatal Surveillance System. Under-reporting of maternal mortality in Canada: a question of definition. Chronic Dis Can. 2002;23(1):22-30.

5. Alkema L, Chou D, Hogan D, Zhang S, Moller AB, Gemmill A, et al; United Nations Maternal Mortality Estimation Inter-Agency Group collaborators and technical advisory group. Global, regional, and national levels and trends in maternal mortality between 1990 and 2015, with scenario-based projections to 2030: a systematic analysis by the UN Maternal Mortality Estimation Inter-Agency Group. Lancet. 2016;387(10017):462-74.

6. World Health Organization (WHO), United Nations International Children's Emergency Fund (UNICEF), United Nations Fund for Population Activities (UNFPA), World Bank Group and the United Nations Population Division. Trends in maternal mortality: 1990 to 2013. Estimates by WHO, UNICEF, UNFPA, World Bank Group and the United Nations Population Division. Disponible en: http: / / apps.who.int/iris / bitstre am/10665/112682/2/9789241507226_eng. pdf Acceso el 15 de diciembre de 2017.

7. Dirección de Estadísticas e Información de Salud. Ministerio de Salud de la Nación. Anuario de Estadísticas Vitales 2013. Argentina 2014. Disponible en: http:/ / www.deis.msal.gov.ar/wp-con$\mathrm{t}$ e $\mathrm{n} \mathrm{t} / \mathrm{u} \mathrm{p}$ lo a d s / $2014 / 12 /$ Serie5Numero57.pdf Acceso el 15 de diciembre de 2017.

8. World Health Organization (WHO), United Nations International Children's Emergency Fund (UNICEF), United Nations Fund for Population Activities (UNFPA), UNICEF, UNFPA, World Bank Group and the United Nations Population Division. Trends in maternal mortality: 1990 to 2015. Estimates by
WHO, UNICEF, UNFPA, World Bank Group and the United Nations Population Division. Disponible en: http:/ / www.who.int/reproductivehealth/publications/monitoring/maternalmortality-2015/en/ Acceso el 15 de agosto de 2017

9. The World Bank/Data/Maternal Mortality Ratio (modeled estimate per 100,000 live births). Disponible en: https:/ / data.worldb a nk. org / indicator / SH.STA. MMRT locations $=\mathrm{AR \& view}=$ chart Acceso el 16 de agosto de 2018.

10. Dirección de Estadísticas de Salud. Ministerio de Salud y Acción Social de la Nación. Sistema Estadístico de Salud. Mortalidad materna en áreas seleccionadas - Año 1985. Buenos Aires; 1992;8(13).

11. Ramos S, Romero M, Karolinski A, Mercer R, Insua I, Rio Fortuna C. Mortalidad materna en la Argentina: diagnóstico para la reorientación de políticas y programas. Centro de Estudios de Estado y Salud. Seminario II, Buenos Aires; 2004.

12. Sociedad de Ginecología y Obstetricia de Buenos Aires (SOGIBA), Dirección General de Estadística y Censos - Gobierno de la Ciudad de Buenos Aires. Omisión de registro de causas de muertes maternas en establecimientos de salud de la Ciudad Autónoma de Buenos Aires. Año 2008. Artículo de investigación. Revista de Obstetricia y Ginecología de Buenos Aires. 2010;89(984):70-84. 
13. Dirección General de Información en Salud. Secretaría de Salud. Búsqueda intencionada de muertes maternas en México. Informe 2008. México, 2010. Disponible en: http:/ / www.dgis.salud.gob.mx/descargas/pdf/BIMM_Informe2008.pdf Acceso el 22 de octubre de 2018.

14. Bouvier-Colle M, Mohangoo A, Gissler M, Novak-Antolic Z, Vutuc C, Szamotulska $\mathrm{K}$, et al for The Euro-Peristat Scientific Committee. What about the mothers? An analysis of maternal mortality and morbidity in perinatal health surveillance systems in Europe. BJOG. 2012;119:880-90.

15. Karimian-Teherani D, Haidinger G, Waldhoer T, Beck A, Vutuc C. Underreporting of direct and indirect obstetrical deaths in Austria, 1980-98. Acta Obstet Gynecol Scand. 2002;81(4):323-7.

16. Torres LM, Rhenals AL, Jiménez A, Ramírez-Villalobos D, Urióstegui R, Piña $\mathrm{M}$, et al. Intentional search and reclassification of maternal deaths in Mexico: The effect on the distribution of causes. Salud Publica Mex. 2014;56:333-47.

17. Cecatti JG, Faúndes A, Surita FG. Maternal mortality in Campinas: evolution, under-registration and avoidance. Sao Paulo Med J. 1999;117(1):5-12.
18. Valongueiro Alves S. Maternal mortality in Pernambuco, Brazil: what has changed in ten years? Reprod Health Matter. 2007;15(30):134-44.

19. Kao S, Chen LM, Shi L, Weinrich MC. Underreporting and misclassification of maternal mortality in Taiwan. Acta Obstet Gynecol Scand. 1997;76(7):629-36.

20. Wu TP, Huang YL, Liang FW, Lu TH. Underreporting of maternal mortality in Taiwan: a data linkage study. Taiwan J Obstet Gynecol. 2015;54(6):705-8.

21. McCaw-Binns AM, Mullings JA, Holder Y. Vital registration and under-reporting of maternal mortality in Jamaica. Int $\mathrm{J}$ Gynaecol Obstet. 2015;128(1):62-7.

22. Secretaría de Salud, Subsecretaría de Integración y Desarrollo del Sector Salud, Dirección General de Información en Salud, Centro Mexicano para la Clasificación de Enfermedades y Centro Colaborador para la Familia de Clasificaciones Internacionales de la OMS en México (CEMECE). Búsqueda Intencionada y Reclasificación de Muertes Maternas -BIRMM- en México. Informe 2012 - 2013. México 2015. Col. Juárez Del. Cuauhtémoc 06600 México, D.F. Disponible en: http://www.dgis. salud.gob.mx/descargas / pdf/
Muertes_Maternas_11_Enero_2016.pdf Acceso el 15 de diciembre de 2017.

23. Tuffin R, Quinn A, Ali F, Cramp P. A review of the accuracy of death certification on the intensive care unit and the proposed reforms to the coroner's system. JICS 2009;10(2):134-7.

24. Rampatige R, Mikkelsen L, Hernandez B, Riley I, Lopez AD. Systematic review of statistics on causes of deaths in hospitals: strengthening the evidence for policy-makers. Bull World Health Organ. 2014;92(11):807-16.

25. Rampatige R, Mikkelsen L, Hernandez B, Riley I, Lopez AD. Hospital cause-ofdeath statistics: what should we make of them? Bull World Health Organ. 2014;92(1):3-3A.

Manuscrito recibido el 11 de junio de 2017. Aceptado para su publicación, tras revisión, el 6 de setiembre de 2018.
SUMMARY

Omission of causes of maternal death in death certificates in Argentina: nationwide observational study
Objective. To determine the magnitude and characteristics of the omission of causes of maternal death in death certificates in Argentina, and to re-estimate the maternal mortality ratio (MMR) for the year 2014.

Methods. Cross-sectional study. Retrospective review of medical records of women of childbearing age who died from causes suspected of concealing maternal deaths in public, social security, and private institutions in all jurisdictions of the country between 1 January and 31 December 2014. The cause of death recorded in the death certificate was reclassified. Outcome measures included: percentage of records with an omission, structure of causes of death, location, time of death with respect to the reproductive process, and gestational age. The RMM was re-estimated on the basis of the results.

Results. Of a sample of 1,176 cases, 969 medical records (82.4\%) were analyzed, identifying 60 cases in which the cause of maternal death was omitted (48 maternal deaths, 12 late maternal deaths). Omissions were found in $14.2 \%$ of maternal deaths and $33.3 \%$ of late maternal deaths. The new estimated MMR for 2014 varied between 43.3 and 47.2 deaths per 100,000 live births.

Conclusions. The omission of causes of maternal death in death certificates in Argentina may be less frequent than international agencies have reported, with differences in omission between regions. Efforts must be made to emphasize the importance of filling out death certificates correctly. 
RESUMO Objetivo. Determinar a dimensão e as características da omissão do registro de causas maternas de morte na Argentina e realizar uma nova estimativa da taxa de mortalidade materna (TMM) para o ano 2014.

\section{Omissão do registro de causas maternas de morte na Argentina: estudo observacional de alcance nacional}

Métodos. Estudo de corte transversal com a revisão retrospectiva dos registros médicos de mulheres em idade reprodutiva que morreram por causas com suspeita de encobrir mortes maternas em instituições da rede pública e previdência social e instituições particulares em todas as jurisdições do país entre $1^{\circ}$. de janeiro e 31 de dezembro de 2014. Foi feita a reclassificação da causa de morte atribuída no Informe Estatístico de Óbitos. As medidas de resultados foram porcentagem de omissão do registro, estrutura de causas de mortes maternas, local, momento da morte em relação ao processo reprodutivo e idade gestacional. Foi realizada uma nova estimativa da TMM com base nos resultados.

Resultados. Foram analisados 969 registros médicos $(82,4 \%)$ de uma amostra de 1.176 casos. Foram identificados 60 casos em que houve omissão da causa materna de morte (48 mortes maternas, 12 mortes maternas tardias). Verificou-se uma porcentagem de omissão de $14,2 \%$ para as mortes maternas e $33,3 \%$ para as mortes maternas tardias. A nova TMM estimada para o ano 2014 oscilou entre 43,3 e 47,2 mortes por 100.000 nascidos vivos.

Conclusões. A omissão de registro de causas maternas de morte na Argentina pode ser menor que a informada pelas agências internacionais. Existe diferença de omissão entre as regiões. São necessários esforços que enfatizem a importância do preenchimento correto da certidão de óbito.

Palavras-chave Sub-registro; morte materna; estatísticas vitais; estudo observacional; Argentina. 